\title{
A Universal Scripting Engine for Transmission Electron Microscopy
}

\author{
James LeBeau, Abinash Kumar and Matthew Hauwiller
}

Massachusetts Institute of Technology, Cambridge, Massachusetts, United States

With the ever increasing need for high quality, statistically significant micro and atomic scale characterization of materials, the workflow of electron microscopy inevitably will require greater instrument control and automation as has been achieved in cryoEM [1-2]. The continued interest in understanding and controlling structure on the nanoscale coupled with the development of materials informatics fields has heightened the demand for reproducible electron microscopy imaging. The current, largely manual nature of materials microscopy data collection can, however, severely limit experiments while slowing down the materials development process. A universal foundation for electron microscopy would empower the next generation of experimental techniques and materials development, and ideally one that is open-source $[3,4]$, accessible, and accessible for every level of microscopist, with or without programming experience.

In this presentation, we will outline the development of a Python-based Universal Scripting Engine for Transmission Electron Microscopy framework that is original equipment manufacturer (OEM) agnostic. We will discuss how the approach helps to automate data acquisition, integrate machine learning, and share complex workflows across platforms. The framework consists of a set of layers, outlined in Figure 1, that link with OEM software to automate data collection, independent of the specific microscope hardware or software. For the greatest degree of flexibility, the framework forms a distributed network, and allows access to control the microscope from Windows, macOS, or Linux. At the instrument level, bridges are used to connect to the microscope and accessories using XML-remote procedure call (RPC) servers. These bridges then communicate through a set of 'techniques' that wrap distinct tasks, such as STEM imaging or microscope optics control, and adopt an abstract base class to ensure that the same workflows can be used regardless of the particular instrument manufacturer. Detectors and holders can also be connected to the framework via the bridges and 'techniques', forming a decentralized system that can interact with every aspect of the data collection.

For the greatest degree of ease-of-use, a workflow-based GUI is provided as a means to rapidly construct new processes on-the-fly to accelerate data collection. The 'work blocks' of the GUI are built to combine techniques and complete sophisticated tasks. This enables non-programmers to build sophisticated workflows in a few clicks, while those experienced with programming can delve into writing bridges, techniques, and work blocks to connect new hardware, add new capabilities, and/or design new experimental protocols. Finally, the USETEM framework is designed to be a community-wide platform for microscopists, and one where we can readily share ideas. To encourage contributions from the community, an auto-citation tool is included that automatically adjusts to the items included in the each workflow. 


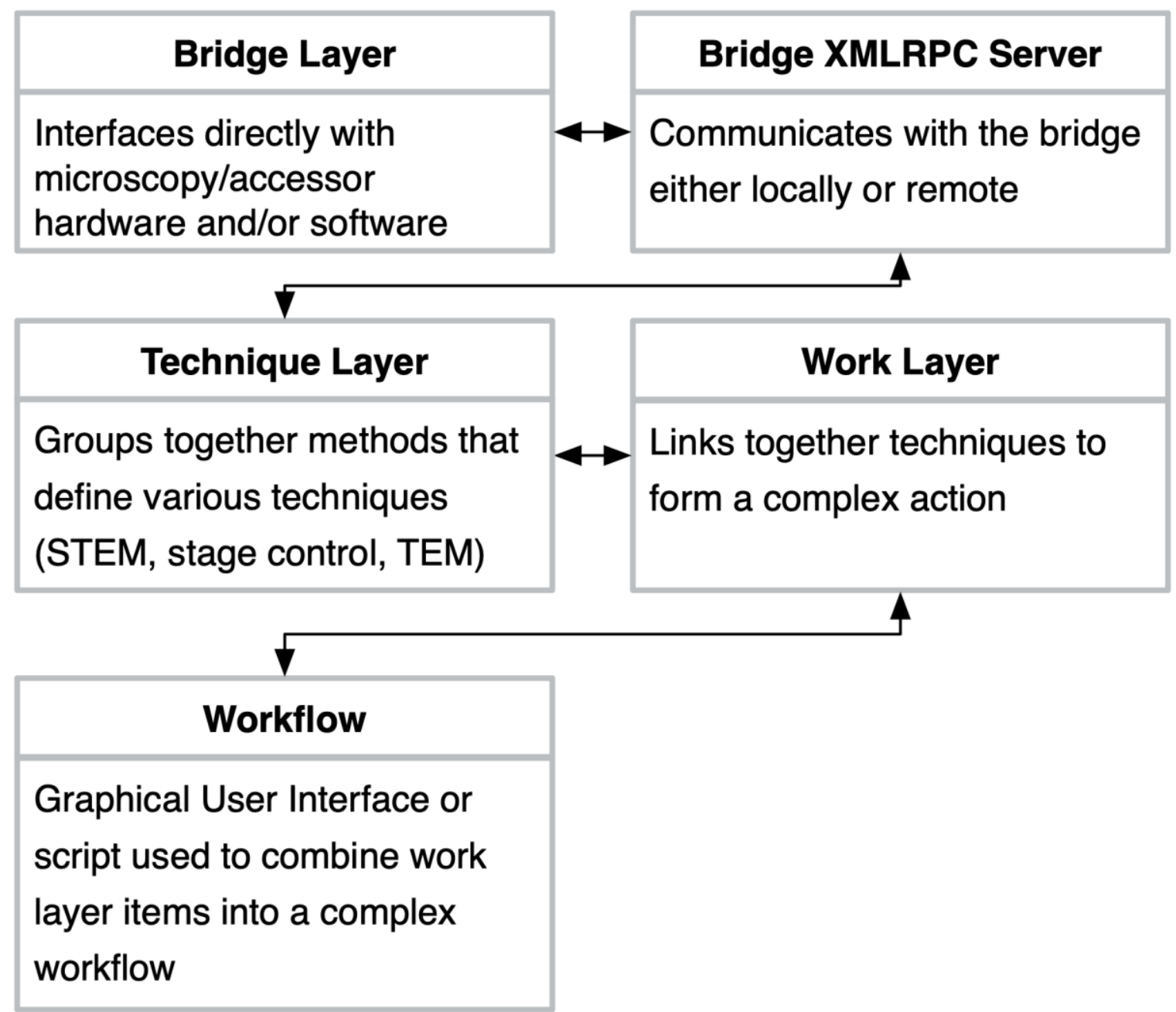

Figure 1. Hierarchical overview of the USETEM framework

\section{References}

[1] Y. Z. Tan, A. Cheng, C. S. Potter, B. Carragher, Automated data collection in single particle electron microscopy, Microscopy 65 (1) (2016) 43-56.

[2] C. Suloway, J. Pulokas, D. Fellmann, A. Cheng, F. Guerra, J. Quispe, S. Stagg, C. S. Potter, B. Carragher, Automated molecular microscopy: The new Leginon system, Journal of Structural Biology 151 (1) (2005) 41-60.

[3] C. Meyer, N. Dellby, J. A. Hachtel, T. Lovejoy, A. Mittelberger, O. Krivanek, Nion Swift: Open Source Image Processing Software for Instrument Control, Data Acquisition, Organization, Visualization, and Analysis Using Python. Microscopy and Microanalysis 25 (S2) (2019) 122-123

[4] S. Somnath, C. R. Smith, S. Jesse, N. Laanait, Pycroscopy - An Open Source Approach to Microscopy and Microanalysis in the Age of Big Data and Open Science, Microscopy and Microanalysis 23 (S1) (2017) 224-225. 